\title{
Novel phonon resonator based on surface screw thread for suppressing thermal transport of Si nanowires
}

\author{
Honggang Zhang,,${ }^{1, *}$ Bo Sun,,${ }^{1, *}$ Song Hu, ${ }^{1}$ Hongyan Wang, ${ }^{1}$ Yajuan Cheng, ${ }^{2}$ Shiyun Xiong, ${ }^{3}$ \\ Sebastian Volz, ${ }^{4,5}$ and Yuxiang Ni॰ ${ }^{1, \dagger}$ \\ ${ }^{1}$ School of Physical Science and Technology, Key Laboratory of Advanced Technologies of Materials, Ministry of Education of China, \\ Southwest Jiaotong University, 610031 Chengdu, People's Republic of China \\ ${ }^{2}$ Key Laboratory of Organic Synthesis of Jiangsu Province and the State and Local Joint Engineering Laboratory for Novel Functional \\ Polymeric Materials, College of Chemistry, Chemical Engineering and Materials Science, Soochow University, Suzhou 215123, \\ People's Republic of China \\ ${ }^{3}$ Functional Nano and Soft Materials Laboratory (FUNSOM) and Collaborative Innovation Center of Suzhou Nano Science and Technology, \\ Soochow University, 215123 Suzhou, People's Republic of China \\ ${ }^{4}$ Laboratoire EM2C, CNRS, CentraleSupélec, Université Paris-Saclay, Grande Voie des Vignes, 92295 Châtenay-Malabry Cedex, France \\ ${ }^{5}$ LIMMS/CNRS-IIS(UMI2820)Institute of Industrial Science, University of Tokyo 4-6-1 Komaba, Meguro-ku, 153-8505 Tokyo, Japan
}

(Received 5 February 2020; revised manuscript received 19 March 2020; accepted 22 April 2020;

published 15 May 2020)

\begin{abstract}
We propose a novel phonon resonator for hindering the thermal transport of nanowires (NWs), based on a screw threadlike helical nanowall. Results from molecular dynamic simulations reveal that the thermal conductivity and phonon transmission of the screw threadlike Si NWs continuously decrease with an increase in the period density of the helical nanowall. The reduction can reach as high as $36 \%$ for the NW with six circles of the helical nanowall, which is much larger than in the case of NWs with straight nanowalls (12\%) and nanopillars (15\%). This phenomenon is due to that the helical nanowall has a larger contact area with the base structure, which leads to a larger volume of the resonating substructure with a constant height and width. Phonon dispersion relations show the formation of flat bands, which confirms the occurrence of phonon resonances due to the surface screw threadlike structures. The phonon spatial distribution reveals mode localization in the helical resonator at the resonant frequency. With regard to suppressing the phonon propagation, the helical nanowall as a phonon resonator exhibits superiority over the straight nanowalls or conventional nanopillars, because (1) it may be easier to fabricate a larger contact area with the base structure and (2) it avoids the problem of nanopillars or nanowalls touching each other, which maintains the ability of generating localized modes. The obtained results provide a novel design of efficient surface phonon resonators to realize nanowires with ultralow thermal conductivity.
\end{abstract}

DOI: 10.1103/PhysRevB.101.205418

\section{INTRODUCTION}

Semiconductor nanowires (NWs) exhibit many attracting properties owing to their confined dimension with nanoscale characteristic lengths [1]. For example, silicon nanowires with diameters on the order of their phonon mean free paths or less show impeded thermal transport due to phonon boundary scattering [2]. The suppressed phonon propagation in NWs is beneficial to applications in thermoelectrics [3], where the energy conversion efficiency is inversely proportional to the thermal conductivity.

A phonon is a collective quantization of the lattice waves. The superposition of phonons of multiple frequencies existing in the solid forms wave packets, which can be considered as particles as long as they are much smaller than the crystal size [4]. Conventionally, the design of low-thermally conducting NWs is based on the phonon particle picture, by utilizing

\footnotetext{
*These authors contributed equally to this work.

†yuxiang.ni@swjtu.edu.cn
}

the phonon scattering mechanisms, such as phonon-boundary scattering [5-7], phonon-imperfection scattering [8-13], and phonon-impurity scattering [14-16]. Phonons with lower frequencies (long wavelengths) will ignore the spatial structural patterns, thus in general only phonons with a relatively high frequency (typically above $1 \sim 2 \mathrm{THz}$ ) can be scattered efficiently. Meanwhile, in most cases, grain boundaries, imperfections, and impurities are unfavorable for the electronic transport, which negates the increase of thermoelectric efficiency.

On the other hand, manipulating the thermal transport based on the wave nature of phonons, particularly by local resonance, has intrigued research interests in recent years [17]. By adding pillars on the base structure, standing waves that hybridize with phonons in the base structure can be introduced, which leads to flat bands in the phonon dispersion relation $[18,19]$. Inspired by this idea, a series of nanophononic metamaterials (NPMs) with resonant structures were proposed to achieve low thermal conductivity. Xiong et al. [20] systematically studied the heat transport in Si based resonant structures. Their molecular dynamic (MD) simulations show 
that the branched NWs can produce numerous resonances in a broad frequency range, and the phonon group velocities were reduced accordingly. Further investigations on oxidized Si membranes show that surface amorphous structures can also produce phonon resonances [21]. Giri et al. used fullerenes as phonon resonators to functionalize carbon nanotubes (CNT) and graphene, and tunable thermal conductivities of individual CNTs and graphene were obtained [22,23]. By introducing phonon resonators, the thermal rectification can also increase up to $470 \%$ under small temperature bias in pristine/branched graphene nanoribbon junctions [24].

Despite the progress made in the thermal transport manipulation with local resonance, further enhancing the impact of the resonators is challenging. It is reported that the phonon transport suppression in Si membranes can be enhanced with increasing volume of the nanopillars, by changing their height and contact area with the main structure [25-27]. However, enlarging the nanopillar size leads to the extreme case of connective nanopillars, which forms a uniform waveguide and favors the thermal transport in the host structure. For example, by replacing the nanopillars by nanowalls (parallel to the phonon transport direction) on a Si membrane, the localized modes almost disappeared and the thermal conductivity reduction decreased [27]. A similar trend was also found for nanopatterned Si membrane systems [28]. In this regard, it is necessary to design a connective resonator structure in order to increase the contact area with the base structure, while maintaining the ability of generating localized modes.

In this work, we propose using a helical nanowall as a phonon resonator for NWs. There are two important advantages of helical nanowall resonators compared with conventional nanopillars or straight nanowalls: (1) The helical nanowall is consecutive, which provides larger contact area with the base structure; (2) the curved nature of the helical nanowall makes it a poor phonon waveguide, which ensures the generation of localized modes. Besides, the screw threadlike NWs are already obtained experimentally in recent years $[29,30]$. Our MD simulations show that, compared with the pristine $\mathrm{Si}$ NW, the screw threadlike Si NW exhibits a $36 \%$ reduction in thermal conductivity $(\kappa)$, much larger than in the case of NWs with straight nanowall (12\%) and nanopillars $(15 \%)$. Phonon analyses such as dispersion relations, group velocity, phonon transmission, and phonon spatial distribution are conducted to gain more insights into the underling physical mechanisms.

\section{METHOD}

\section{A. Computational models}

The atomistic models of $\mathrm{Si}$ nanowires are illustrated in Fig. 1. Cylindrical Si NWs are obtained by cleaving the bulk Si supercells along the [100] direction. The radius of the cross section $(1.48 \mathrm{~nm})$ and the total length $(38 \mathrm{~nm})$ are fixed in all the NW models. Helical nanowall, i.e., the screw thread, is then added to the main NWs. We label the NWs according to the period number of the helical nanowall on NWs with a fixed length (38 nm). For example, $2 \mathrm{P}$ corresponds to the NW with two circles of the helical nanowall [Fig. 1(b)], while 0P refers to the pristine NW with the same length [Fig. 1(a)]. To investigate the impact of the period density of helical nanowall

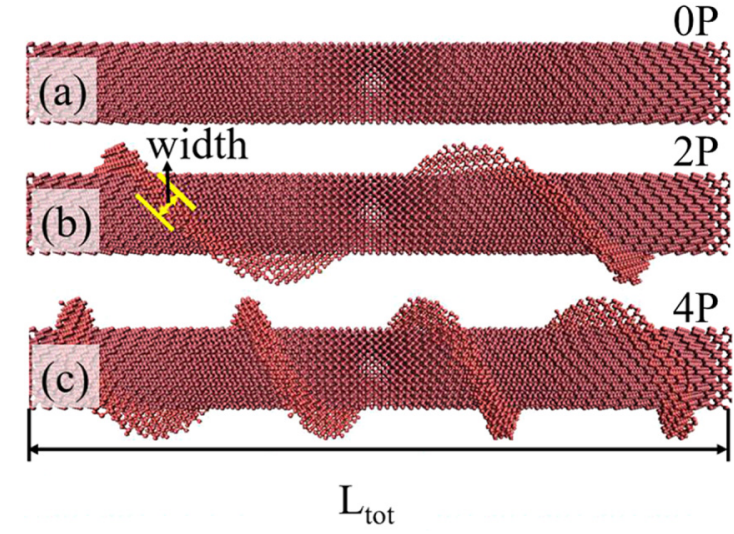

FIG. 1. Atomistic models of Si nanowires: (a) pristine, and (b),(c) with helical nanowall.

on the thermal transport of NWs, we design a set of NWs from $1 \mathrm{P}$ to $6 \mathrm{P}$. The width of the helical nanowall is $0.32 \mathrm{~nm}$ and the height is set between 0.5 and $3.5 \mathrm{~nm}$.

\section{B. Thermal conductivity calculation of screw threadlike Si NWs}

We compute the thermal conductivity $(\kappa)$ along the NWs by using the nonequilibrium molecular dynamics (NEMD) with the LAMMPS package [31]. The Stillinger-Weber (SW) potential is employed to describe the covalent $\mathrm{Si}-\mathrm{Si}$ bond [32]. Free boundary conditions are applied at the boundaries transverse to the direction of the heat current flow, while atoms at both ends of the structures are fixed. Adjacent to the two fixed areas, sections with a length $L_{\text {bath }}=10 \mathrm{~nm}$ are set as the hot and cold heat baths, as illustrated in Fig. 2. To reach the equilibrium state, a 500 ps simulation run is performed in the canonical ensemble (NVT) at $300 \mathrm{~K}$, followed by a 200 ps run in the microcanonical ensemble (NVE), with a time step of $1 \mathrm{fs}$. Subsequently, the Langevin thermostat is applied to the groups of atoms in the thermal baths to achieve temperatures $T_{\text {hot }}=T+\Delta T$ and $T_{\text {cold }}=T-\Delta T$, respectively. Here $T$ is set to $300 \mathrm{~K}$ and $\Delta T$ is $60 \mathrm{~K}$. A 2 ns run was performed to reach the steady state and another $8 \mathrm{~ns}$ run to time average the local temperature and heat flux along the wires. The thermal conductivity $\kappa$ along the wires is calculated from the temperature gradient $\nabla T$ and the heat flux $Q / S$ determined from a steady state according to Fourier's Law:

$$
\kappa=\frac{Q}{S \nabla T} .
$$

Here, $Q$ is the thermal power across a cross-sectional area $S$ perpendicular to the transport direction. It should be noted that both the cross-sectional area of the NW and the helical

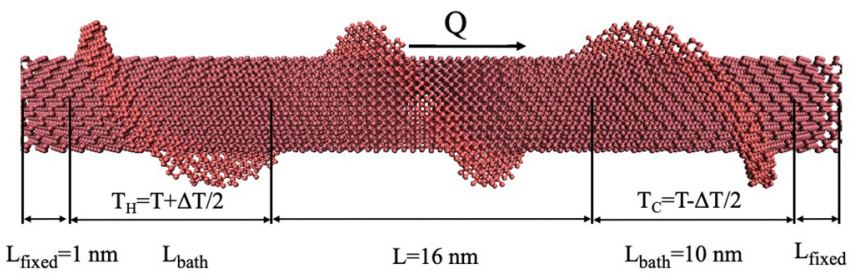

FIG. 2. Schematic illustration of a Si nanowire model (3P) for NEMD simulations. 
nanowall are included in $S$. In our study, the size (length) effect in NEMD is not removed in the computations, since our purpose is to compare the trend of the relative $\kappa$ change rather than to obtain the absolute value of the thermal conductivity.

\section{Phonon transmission spectra calculations}

To uncover the physical mechanism of the phonon transport in our models, phonon analyses were performed based on MD simulations. We use the same NEMD simulation set to calculate the phonon transmission spectra $[\operatorname{Trans}(\omega)]$, by using the method developed by K. Saaskilahti et al. $[33,34]$. The frequency resolved heat flux between any atoms $i$ and $j$ can be expressed as:

$$
q_{i \rightarrow j} \approx-\frac{2}{t_{\mathrm{simu}} \omega} \sum_{\alpha, \beta \in\{x, y, z\}} \operatorname{Im}\left\langle\hat{v}_{i}^{\alpha}(\omega)^{*} K_{i j}^{\alpha \beta} \hat{v}_{j}^{\beta}(\omega)\right\rangle,
$$

where $t_{\text {simu }}$ and $\omega$ are the simulation time and the frequency, respectively. $\hat{v}_{i}^{\alpha}$ and $\hat{v}_{j}^{\beta}$ are the Fourier transformed atomic velocities of atom $i$ in direction $\alpha$ and atom $j$ in direction $\beta$, respectively. For calculating the flux spectra, the force constant matrix is calculated with the finite displacement method by Lammps, i.e., after relaxing the structures to the potential minimum, atom $i$ is moved in the directions $\pm x, \pm y$, and $\pm z$ with a small value ( $\Delta=0.01 \AA$ ). After each displacement, the forces $F$ of each atoms are calculated. As a result, the element of the force constant matrix can be evaluated as:

$$
K_{i j}^{\alpha \beta}=\frac{F_{j}^{\beta-}-F_{j}^{\beta+}}{2 \Delta},
$$

here $F_{j}^{\beta-}$ and $F_{j}^{\beta+}$ denote the force on atom $j$ in $\beta$ direction when atom $i$ is displaced to $-\alpha$ and $+\alpha$ directions, respectively. The heat current across any interface separating adjacent atom sets $L$ and $R$ can be obtained by summing over atoms in each set:

$$
q(\omega)=\sum_{i \in L} \sum_{j \in R} q_{i \rightarrow j}(\omega) .
$$

The phonon transmission function can thus be defined according to the spectral heat current as:

$$
\operatorname{Trans}(\omega)=\frac{q(\omega)}{k_{B} \Delta T},
$$

where $\Delta T$ is the temperature difference of the two thermal baths in NEMD simulations.

\section{Phonon dispersion relation, group velocity, and spatial distribution calculations}

The phonon dispersion relations and the group velocity of the nanowires were obtained based on the harmonic lattice dynamics [35]. The normal-mode eigenvector components $\varepsilon$ and their corresponding eigenfrequencies $\omega$ were obtained by solving the lattice dynamics equation,

$$
D(k) \varepsilon=\omega^{2} \varepsilon .
$$

$D(k)$ is the atomic mass normalized dynamical matrix, which is calculated from the second derivative of the Stillnger-Weber potential used in MD simulations. One period of the NW with helical nanowall (the repeating unit) was chosen as the unit cell in our calculations. Phonon group velocity was then derived according to the dispersion relations.

The spatial distribution of the phonon modes within a given frequency range of the models was calculated as follows [24]:

$$
\phi_{i, \omega}=\frac{\sum_{\omega \in \Lambda} \sum_{\alpha} \varepsilon_{i \alpha, \omega}^{*} \varepsilon_{i \alpha, \omega}}{\sum_{j} \sum_{\omega \in \Lambda} \sum_{\alpha} \varepsilon_{i \alpha, \omega}^{*} \varepsilon_{i \alpha, \omega}},
$$

where $\alpha$ and $i$ correspond to the Cartesian component $(x, y$, or $z$ ) and the atom index, respectively. $\Lambda$ denotes a frequency range and we considered $0.25 \pm 0.2 \mathrm{THz}$ and $14.5 \pm 0.2 \mathrm{THz}$ in this study.

\section{RESULTS AND DISCUSSION}

\section{A. Thermal conductivity and phonon transmission spectra of screw threadlike Si NWs}

Figure 3(a) shows the calculated thermal conductivity $(\kappa)$ of the screw threadlike Si NWs as a function of different period density of helical nanowall at $300 \mathrm{~K}$. It can be seen that the screw threadlike surface has obvious impact on the thermal conductivity of Si NWs. $\kappa$ decreases from $6.7 \mathrm{~W} / \mathrm{mK}$ (pristine) to $5.5 \mathrm{~W} / \mathrm{mK}(1 \mathrm{P})$. With the increasing period density of the helical nanowalls, $\kappa$ continuously decreases, suggesting that a larger contact area of the base structure and the resonator is more efficient in suppressing the phonon propagation. A $35 \%$ reduction in $\kappa$ is obtained for $6 \mathrm{P} \mathrm{NW}$ compared with the pristine one. We attribute the thermal transport impedance in the screw threadlike Si NWs to the standing waves generated by the helical nanowalls that hybridize with the phonons (especially with low frequency) in the base NW. Evidences from the phonon dispersion relations and the group velocity will be shown later in the following section. It is interesting to note that by changing the atomic type of the helical nanowall from $\mathrm{Si}$ to $\mathrm{Ge}, \kappa$ can be further decreased from $5.4 \mathrm{~W} / \mathrm{mK}$ to $4.7 \mathrm{~W} / \mathrm{mK}$.

To have a deeper understanding of the $\kappa$ reduction, we calculated the phonon transmission spectra, as shown in Fig. 3(b). Overall, the phonon transmission spectrum decreases with increasing period density of the helical nanowall, which complies well with the thermal conductivity results. It should be noted that, for $4 \mathrm{P}$ and $6 \mathrm{P}$ models, there is obvious decrease of the transmission spectra in the low-frequency regime (below $4 \mathrm{THz}$ ), indicating the importance of the resonant effect at low frequencies. Such phenomenon was not observed in our previous studies on phonon-imperfection scattering [13] and phonon-impurity scattering [36], in which cases the transmission spectra almost remain unaltered in the low-frequency regime, and the ballistic transport is obvious due to the long-wavelength nature of these phonons. When the atomic type of the helical nanowall is changed from $\mathrm{Si}$ to $\mathrm{Ge}$, the corresponding phonon transmission is further suppressed in the high frequency range (above $4 \mathrm{THz}$ ), as shown in the inset of Figure 3(b). This result implies that, to further suppress the phonon transport, the phonon resonance can be combined with other phonon hindering mechanisms such as introducing acoustic impedance.

We now evaluate the impact of the height and width of the helical nanowall on the NW thermal conductivity. As demonstrated in Fig. 4, $\kappa$ monotonically decreases with the 

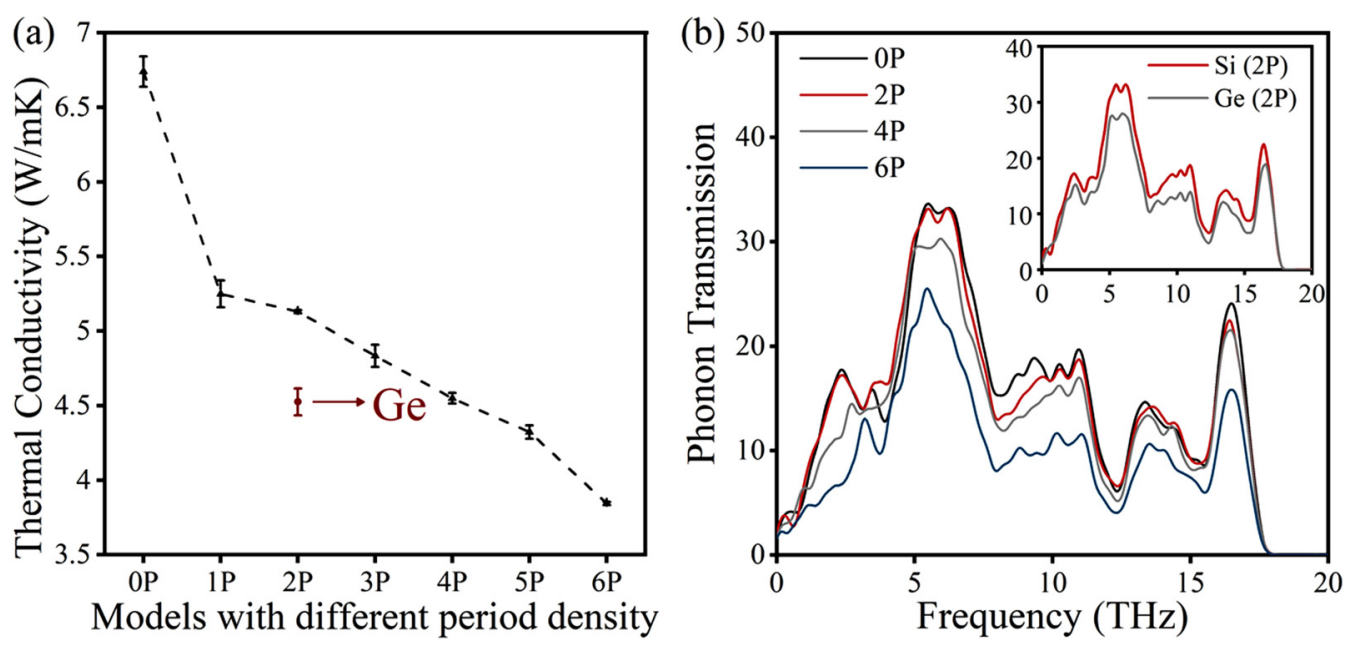

FIG. 3. (a) Thermal conductivity of Si NWs varying with the period density of the helical nanowall. The red dot indicates the thermal conductivity of the model with Ge helical nanowall (the main NW is still Si). The height of the nanowall is set to $1 \mathrm{~nm}$. (b) Phonon transmission spectra of Si NWs with different period density of the helical nanowall. The inset compares the transmission spectra of the models with Si and Ge nanowalls, respectively.

height of the screw thread. This phenomenon is expected because taller nanowall exhibits more local resonance modes (vibrons) than a thinner one and therefore more strongly affects the phonon propagation for a given length of NW section. On the other hand, $\kappa$ is not sensitive to the width of the helical nanowall, as shown in Table I. This phenomenon may be due to the competition between the increased room for phonon propagation and the increased number of localized modes.

\section{B. Analyses of phonon dispersion relation, group velocity, and spatial distribution}

To confirm the phonon resonance effect of the helical nanowalls, we compare the phonon dispersion relations of pristine Si NW and 1P screw threadlike Si NW in Figs. 5(a) and 5(b). It can be obviously seen that, for the screw threadlike Si NW, a series of resonant flat bands crossing the entire Brillouin zone are generated. For example, the lowest resonant frequency locates around $0.25 \mathrm{THz}$, where the coupling be-

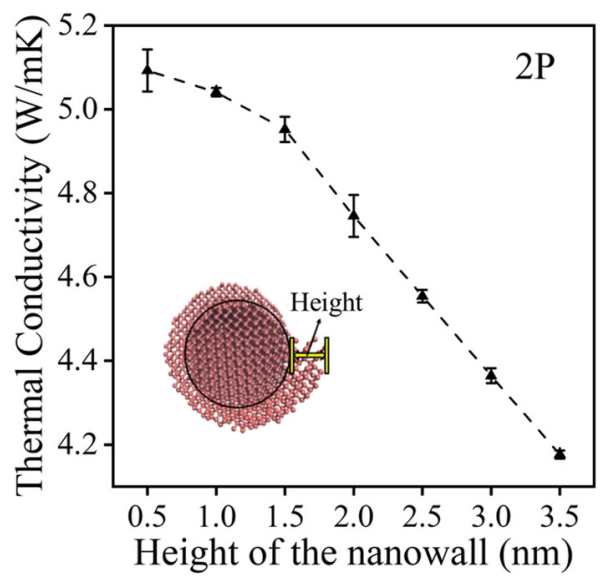

FIG. 4. The thermal conductivity of Si NWs against the height of the helical nanowall.
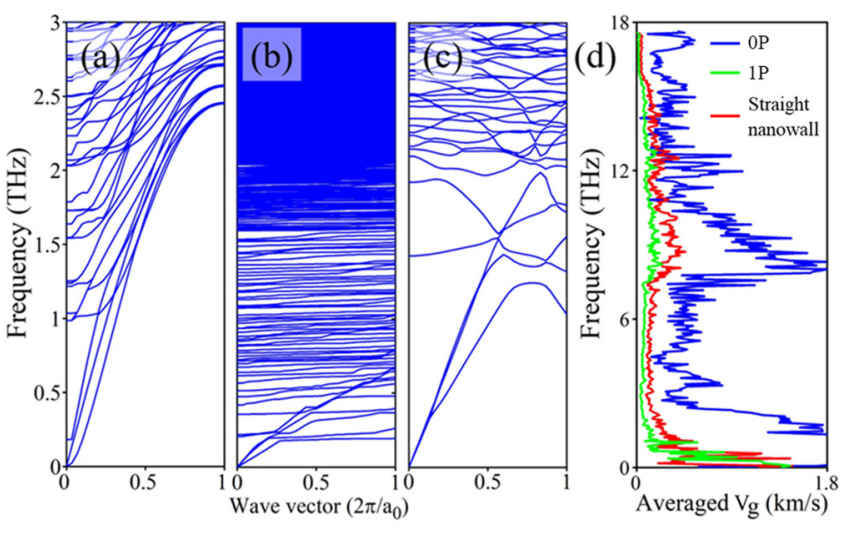

$\omega=0.25 \mathrm{THz}$
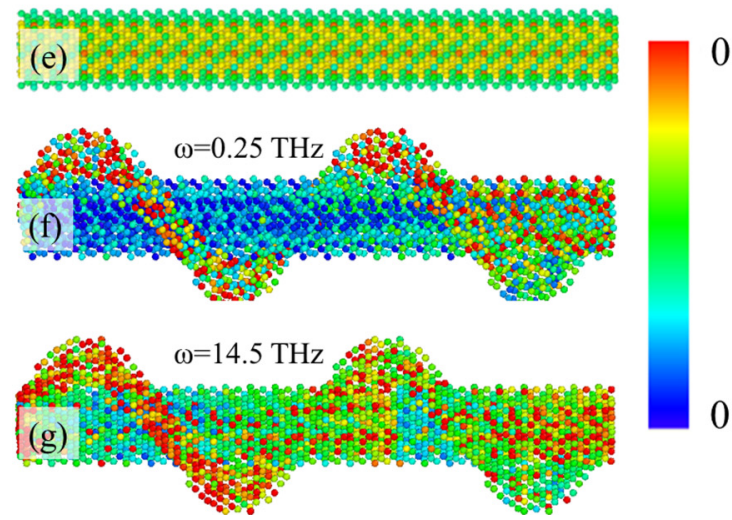

FIG. 5. (a)-(c) Phonon dispersion relations in the frequency range $[0,3] \mathrm{THz}$ for the pristine $\mathrm{Si} \mathrm{NW}, 1 \mathrm{P}$ screw threadlike Si NW, and Si NW with surface straight nanowall, respectively. (d) Averaged phonon group velocities of the different models. (e)-(g) Spatial distribution of the modes in the pristine and the screw threadlike NWs with the resonant frequency $(\omega=0.25 \mathrm{THz})$ and ordinary frequency $(\omega=14.5 \mathrm{THz})$. 
TABLE I. Thermal conductivity of Si NWs against the width of the helical nanowall (2P).

\begin{tabular}{llll}
\hline \hline Width $(\mathrm{nm})$ & 0.32 & 0.64 & 0.96 \\
\hline$\kappa(\mathrm{W} / \mathrm{mK})$ & 5.1 & 5.0 & 5.2 \\
\hline \hline
\end{tabular}

tween the LA mode and the resonant phonon can be observed. The standing waves that hybridize with the low-frequency phonons in the main NW lead to flat bands in the phonon dispersion relation. Therefore, sizable reduction is observed for the phonon group velocity of the screw threadlike $\mathrm{Si}$ NW, compared with that of the pristine Si NW, as shown in Fig. 5(d).

To have an intuitive image of the phonon resonance, we calculated the spatial distribution of phonon modes from Eq. (7). According to the phonon dispersion relations, $0.25 \mathrm{THz}$ is a resonant frequency, while $14.5 \mathrm{THz}$ is an ordinary frequency where no phonon resonance occurs. For the NWs with helical nanowall, we calculated the phonon spatial distribution of the modes within the frequency ranges of $0.25 \pm 0.2 \mathrm{THz}$ and $14.5 \pm 0.2 \mathrm{THz}$, as illustrated in Figs. 5(f) and 5(g). The phonon spatial distribution of a pristine NW is also calculated for comparison [Fig. 5(e)]. Here, the range $\pm 0.2 \mathrm{THz}$ is considered to ensure the inclusion of the resonant modes (if any) around the corresponding frequency. The color bar indicates the portion of the local mode energy over the total energy, at a given frequency range. Figure 5(e) shows that phonons distribute uniformly in the pristine NW. When the helical nanowall is added, phonons with the resonant frequency are mostly localized in the helical nanowall, and only a few atoms in the main NW participate in the vibration [Fig. 5(f)]. In contrast, Fig. 5(g) shows that the spatial distribution of an ordinary mode (around $14.5 \mathrm{THz}$ ) is relatively uniform, indicating that the phonon vibration is not affected by the resonators at this frequency.

\section{Comparison between the impacts of helical and straight nanowalls on NW thermal conductivity}

In this section, from two aspects, we will highlight the advantages of using helical nanowall as phonon resonators to suppress the phonon propagation in NWs. Firstly, we compare the thermal conductivity of Si NWs with resonators of helical and straight nanowalls, respectively, in order to show the advantage of the curved nature of the resonator. Secondly, we evaluate the impact of the continuity of the nanowalls on the NW thermal conductivity. Computational models are built as demonstrated in Fig. 6. Helical (3P) and straight nanowalls are introduced to Si NW [Figs. 6(a) and 6(c)], and then a set of atoms is removed from the nanowalls, leaving nanowall sections (nanopillars) with equal spaces [Figs. 6(b) and 6(d)]. Note that the straight nanowall is placed along the NW axis, which is parallel to the heat transport direction under consideration.

Figure 7 shows the thermal conductivity of Si NWs with resonators of helical and straight nanowalls as a function of the volume fraction $\left(n_{v}\right)$ of the removed atoms. At $n_{v}=0$, both straight and helical nanowalls are consecutive, and $\kappa$ of the helical nanowall case is about $10 \%$ smaller than that

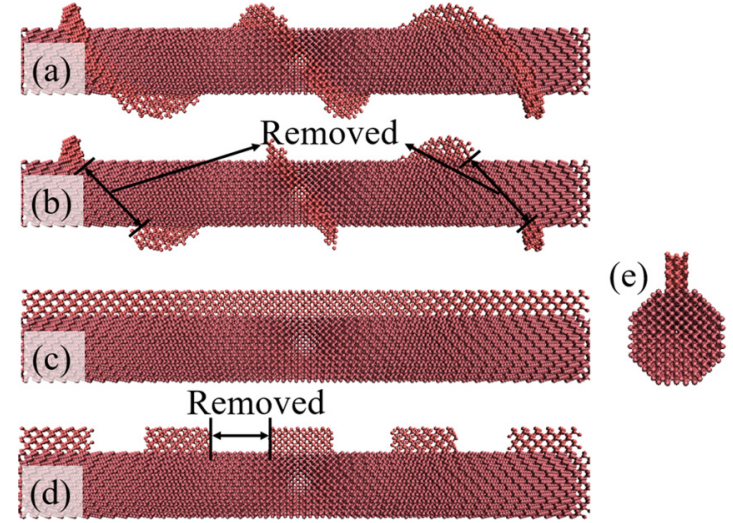

FIG. 6. Models of Si NWs with (a) helical (3P) and (c) straight nanowalls, and the corresponding NWs (b) and (d) with nanowall sections with equal spaces after the removal of certain atoms. (e) Side view of the NW with straight nanowall.

of the straight one. This discrepancy can be due to the fact that a large part of the localized modes in NWs with straight nanowall disappeared, as shown in Fig. 5(c). The dispersion diagram of Si NW with straight nanowall is relatively close to that of the pristine NW [Fig. 5(a)]. In this case, the straight nanowall is a uniform waveguide along the axial direction [27]. Phonon resonance would not occur without the localized modes, and therefore, the thermal conductivity is larger. When the nanowall revolves around the nanowire, phonon propagation along the curved nanowall is more difficult, and localized modes can be generated and then couple with the low-frequency modes in the main wire [Fig. 5(b)]. In this regard, the curved nature of the nanowall is important for phonon resonators.

As $n_{v}$ increases, in both straight and helical cases, $\kappa$ first slightly decreases and then increases and finally converges to $n_{v}=1$ (the pristine NW). The slight decrease in $\kappa$ stems from the breakdown of the nanowall continuity, which blocks the phonon transmission along the nanowall. As more atoms are removed, the contact area between the resonators and the host

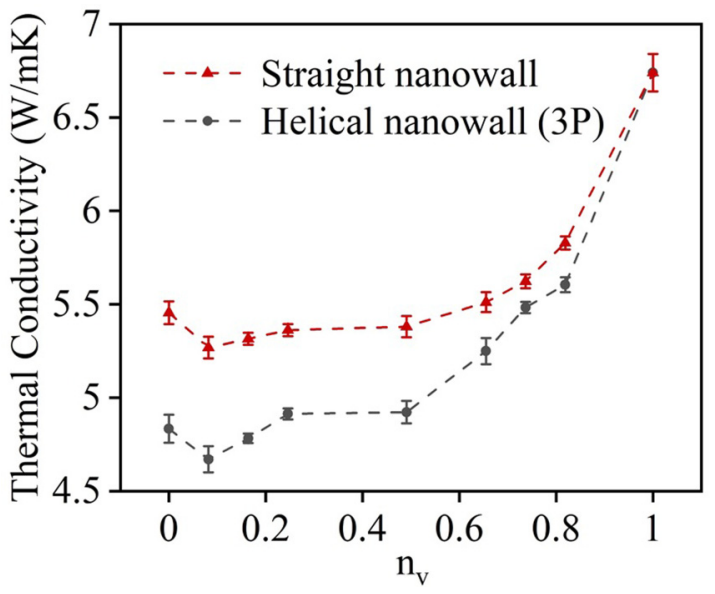

FIG. 7. The thermal conductivity of Si NWs with resonators of helical and straight nanowalls as a function of the volume fraction of the removed atoms. 
NW decreases, and the resonator impact on $\kappa$ is weakened, which leads to the $\kappa$ increase. It can be clearly seen that $\kappa$ of NWs with helical nanopillars is still lower than that of the straight nanopillar cases, owing to the larger volumetric size of the helical nanopillars relative to the straight ones at the same $n_{v}$. As $n_{v}$ further grows, the difference in $\kappa$ between the two cases gets smaller, until $\kappa$ of both cases converge to the value of the pristine NW. The results revealed by Fig. 7 prove that the helical resonators are more beneficial for suppressing the phonon transport than conventional nanopillars.

\section{CONCLUSIONS}

In this study, we propose using the helical nanowall as a resonator for nanowires. Our molecular dynamics simulations show that, compared with the pristine $\mathrm{Si} \mathrm{NW}$, the screw threadlike Si NW exhibits a $36 \%$ reduction in thermal conductivity (6P), much larger than in the case of NWs with a straight nanowall $(12 \%)$ and nanopillars $(15 \%)$. Phonon transmission spectra show the hindering of phonon transport in the whole frequency range. Phonon analysis such as dispersion relations, group velocity, and phonon spatial distribution confirm the phonon resonance effect in the screw threadlike Si nanowires. We conclude that the helical nanowall as a phonon resonator exhibits superiority over the straight nanowall or conventional nanopillars, owing to its large contact area with the base structure while maintaining the ability of generating localized modes. The radius of NWs $(1.48 \mathrm{~nm})$ and the height of the nanowall $(0.5-3.5 \mathrm{~nm})$ in our theoretical models can be scaled to the experimentally synthesized screw threadlike NWs $(5 \mathrm{~nm}$ for both the NW radius and the height of the nanowall [29]). Therefore, the findings in this study should be valuable to the design of efficient surface phonon resonators for nanowires, which is important for their applications in thermoelectrics and thermal insulators, where low thermal conductivity is desired.

\section{ACKNOWLEDGMENTS}

Y.N. acknowledges the support of National Natural Science Foundation of China (NSFC) Grant No. 11774294, and the R\&D Program for International S\&T Cooperation and Exchanges of Sichuan province (Grant No. 2018HH0088). S.X. acknowledges the financial support from National Natural Science Foundation of China (Grant No. 11804242). This work is also supported by the Fundamental Research Funds for the Central Universities (2682019CX67). The authors thank Prof. Jie Chen from Tongji University for useful discussions.
[1] G. S. Doerk, C. Carraro, and R. Maboudian, ACS Nano 4, 4908 (2010).

[2] D. Li, Y. Wu, P. Kim, L. Shi, P. Yang, and A. Majumdar, Appl. Phys. Lett. 83, 2934 (2003).

[3] A. I. Hochbaum, R. Chen, R. D. Delgado, W. Liang, E. C. Garnett, M. Najarian, A. Majumdar, and P. Yang, Nature (London) 451, 163 (2008).

[4] G. Chen, Nanoscale Energy Transport and Conversion: A Parallel Treatment of Electrons, Molecules, Phonons and Photons (Oxford University Press Inc, New York, 2005).

[5] M. M. Rojo, B. Abad, C. V. Manzano, P. Torres, X. Cartoixa, F. X. Alvarez, and M. M. Gonzalez, Nanoscale 9, 6741 (2017).

[6] G. Xie, Y. Guo, B. Li, L. Yang, K. W. Zhang, M. Tang, and G. Zhang, Phys. Chem. Chem. Phys. 15, 14647 (2013).

[7] J. Mao, Y. Wang, Z. Liu, B. Ge, and Z. Ren, Nano Energy 32, 174 (2017).

[8] Y. Ni, S. Xiong, S. Volz, and T. Dumitrică, Phys. Rev. Lett. 113, 124301 (2014).

[9] K. F. Murphy, B. Piccione, M. B. Zanjani, J. R. Lukes, and D. S. Gianola, Nano Lett. 14, 3785 (2014).

[10] S. Xiong, J. Ma, S. Volz, and T. Dumitrică, Small 10, 1756 (2014)

[11] P. N. Martin, Z. Aksamija, E. Pop, and U. Ravaioli, Phys. Rev. Lett. 102, 125503 (2009).

[12] J. Chen, G. Zhang, and B. Li, Nano Lett. 10, 3978 (2010).

[13] S. Hu, H. Zhang, S. Xiong, H. Zhang, H. Wang, Y. Chen, S. Volz, and Y. Ni, Phys. Rev. B 100, 075432 (2019).

[14] N. Yang, G. Zhang, and B. Li, Nano Lett. 8, 276 (2008).

[15] H. Zhang, H. Han, S. Xiong, H. Wang, S. Volz, and Y. Ni, Appl. Phys. Lett. 111, 121907 (2017).

[16] Y. Pan, G. Hong, S. N. Raja, S. Zimmermann, M. K. Tiwari, and D. Poulikakos, Appl. Phys. Lett. 106, 093102 (2015).
[17] M. I. Hussein, C. Tsai, and H. Honarvar, Adv. Funct. Mater. 30, 1906718 (2020).

[18] B. L. Davis and M. I. Hussein, Phys. Rev. Lett. 112, 055505 (2014).

[19] H. Honarvar and M. I. Hussein, Phys. Rev. B 93, 081412(R) (2016).

[20] S. Xiong, K. Saaskilahti, Y. A. Kosevich, H. Han, D. Donadio, and S. Volz, Phys. Rev. Lett. 117, 025503 (2016).

[21] S. Xiong, D. Selli, S. Neogi, and D. Donadio, Phys. Rev. B 95, 180301(R) (2017).

[22] A. Giri and P. E. Hopkins, Phys. Rev. B 98, 045421 (2018).

[23] A. Giri and P. E. Hopkins, J. Appl. Phys. 125, 205102 (2019).

[24] X. Chen, J. Liu, Z. Xie, Y. Zhang, Y. Deng, and K. Chen, Appl. Phys. Lett. 113, 121906 (2018).

[25] H. Honarvar, L. Yang, and M. I. Hussein, Appl. Phys. Lett. 108, 263101 (2016).

[26] Z. Wei, J. Yang, K. Bi, and Y. Chen, J. Appl. Phys. 118, 155103 (2015)

[27] H. Honarvar and M. I. Hussein, Phys. Rev. B 97, 195413 (2018).

[28] S. Neogi and D. Donadio, Eur. Phys. J. B 88, 73 (2015).

[29] N. Zhang, L. Bu, S. Guo, J. Guo, and X. Huang, Nano Lett. 16, 5037 (2016)

[30] J. Tang, Q. Chen, L. You, H. Liao, S. Sun, S. Zhou, Z. Xu, Y. Chen, and G. Guo, J. Mater. Chem. A 6, 2327 (2018).

[31] S. Plimpton, J. Comput. Phys. 117, 1 (1995).

[32] F. H. Stillinger and T. A. Weber, Phys. Rev. B 31, 5262 (1985).

[33] K. Sääskilahti, J. Oksanen, J. Tulkki, and S. Volz, Phys. Rev. B 90, 134312 (2014).

[34] K. Sääskilahti, J. Oksanen, S. Volz, and J. Tulkki, Phys. Rev. B 91, 115426 (2015).

[35] H. Zhao and J. B. Freund, J. Appl. Phys. 97, 024903 (2005).

[36] Y. Ni, H. Zhang, S. Hu, H. Wang, S. Volz, and S. Xiong, Int. J. Heat Mass Transf. 144, 118608 (2019). 